\title{
EL ROL DE LOS MODELOS MENTALES ESPACIALES EN LA APREHENSIÓN LITERARIA ${ }^{1}$
}

\section{THE ROLE OF SPATIAL MENTAL MODELS IN THE LITERARY COMPREHENSION}

\author{
Roy Alfaro Vargas*
}

... one cannot talk about events in the universe without the notions of space and time...

\section{A Brief History of Time}

Stephen Hawking

\begin{abstract}
RESUMEN
Este artículo analiza la relación espacio-tiempo dentro de los procesos de conformación de los modelos mentales espaciales. También, se critica la posición sostenida al respecto por la psicología cognitiva. De igual modo, se establece una relación entre los modelos mentales y las concepciones físicas sostenidas por Stephen Hawking, dentro de un planteamiento dialéctico, con el fin de plantear el concepto de modelos mentales tempo-espaciales.
\end{abstract}

PALABRAS CLAVE: PSICOLOGÍA COGNITIVA * SOCIOLOGÍA LITERARIA * TEORÍA LITERARIA $*$ MODELOS MENTALES $*$ TIEMPO $*$ ESPACIO

\section{ABSTRACT}

This article analyzes the relation existing between time and space, in the conformation of spatial mental models. Besides, it criticizes the cognitive proposal developed by the cognitive psychology. Also, it sets up a relation between the mental models and the Stephen Hawking's physical conceptions in a dialectical perspective so that it defines the time-spatial mental models' concept.

KEYWORDS: COGNITIVE PSYCHOLOGY $*$ SOCIOLOGY OF LITERATURE $*$ LITERARY THEORY $*$ MENTAL MODELS $*$ TIME $*$ SPACE

$1 \quad$ Un agradecimiento al Dr. William Langston (1998) por ayudarme a acceder a su texto. De igual modo, es preciso agradecer al Lic. Gonzalo Mora Chavarría por su paciencia en la explicación de los conceptos matemáticos y físicos que aquí se discuten.
Candidato a la Maestría Académica en Literatura Latinoamericana, Universidad de Costa Rica. royalfarov@costarricense.cr 


\section{INTRODUCCIÓN}

En la literatura, el espacio siempre juega un rol fundamental. Los personajes se ubican dentro de una representación (Vorstellung) que está ligada al campo, la ciudad, una habitación $o$, inclusive, a la representación mental de un espacio imaginario.

El espacio se liga tanto al marco en donde el personaje actúa, como al desplazamiento que él ejecuta al caminar en la ciudad o entre los objetos de una habitación. El espacio puede absorber todo el valor simbólico de una trama. Por ejemplo, La metamorfosis de Kafka refiere a un espacio habitacional cerrado, pequeño e incómodo, que remite a la alienación sufrida en las sociedades capitalistas, en donde el ser humano no tiene espacio para desarrollarse de manera íntegra y no-monótona. El espacio puede representar el elemento de cambio en la perspectiva existenciaria de un personaje, como en el caso de Los pasos perdidos de Alejo Carpentier, en donde el cambio del espacio urbano, por un espacio primitivo, permite al personaje principal reelaborar su identidad.

El espacio textual, sin embargo, tiene su corolario material, es decir, el espacio físico, el espacio de la cotidianidad, en donde el lector ejecuta su praxis. Este espacio práxico se encuentra en estrecha correlación con el espacio de la representación espacial en la literatura.

Dentro de esta perspectiva general, la cual luego será argumentada debidamente, nosotros planteamos la siguiente tesis: la representación del espacio en el texto se inserta dentro de un modelo mental caracterizado como un espacio euclidiano, el cual se inserta, a la vez, dentro de una noción físico-mecánica (física newtoniana) la cual responde a un proceso de disonancia cognitiva que niega la indisoluble relación tiempo-espacio, más ligada a la física relativista $y$, en nuestro caso, a las propuestas de Hawking; $y$ que, por tanto, tal disonancia se establece como un medio positivo de control social.

Para llevar a buen puerto nuestra tesis, es preciso cumplir con los siguientes objetivos:

1. Definir el concepto de modelo mental.

2. Determinar el desarrollo de la aplicación del concepto de modelo mental al estudio del espacio, dentro del ámbito de la aprehensión textual, en la psicología cognitiva.

3. Establecer las limitaciones de la relación espacio-aprehensión textual, en relación con el enfoque psicológico cognitivo.

4. Identificar el tipo de espacio ligado a los estudios de los modelos mentales, en la psicología cognitiva.

5. Señalar la relación indisoluble tiempoespacio, como un medio para superar la espacialidad de los estudios de los modelos mentales de la psicología cognitiva.

6. Definir la transducción, como premisa teórica-metodológica, en donde es posible reestablecer una relación de la unidad dialéctica tiempo-espacio.

\section{LOS MODELOS MENTALES: UNA DEFINICIÓN}

Lo primero que debemos hacer es definir el concepto de modelo mental. Tal concepto refiere a "una representación de una situación, la cual contiene elementos que representan gente, cosas, eventos, estados, procesos, tiempos y lugares; las cuales establecen relaciones reales entre ellos" (Grarnham, 1993)2.

Es decir, un modelo mental es un mapa contextual, en donde el individuo interioriza como representación, una situación determinada. O sea, el modelo mental "es una representación acerca de lo que el lenguaje es, más que una representación del lenguaje mismo" (Langston, 1998: 247) ${ }^{3}$. Por ende, un modelo mental es un concepto que remite a la referencialidad del lenguaje, más que a una relación significante/significante.

De alguna manera, el concepto de modelo mental, tal y como lo definen los psicólogos cognitivos, se aleja de la concepción simbólica del pensamiento (CSP), ya que, para tales concepciones simbólicas, un significante siempre refiere a otro significante, con lo cual el símbolo y la representación adquieren un estatus de igualdad. Con esto, "La significatividad (meaningfulness) de los

$\begin{array}{ll}2 & \text { Traducción nuestra. } \\ 3 & \text { Traducción nuestra. }\end{array}$


símbolos [que] es necesariamente derivativa de la existencia del pensamiento" (Kuczynski, 2005: 245) ${ }^{4}$ impide que podamos considerar al pensamiento como un proceso operado por símbolos (Kuczynski, 2005: 245). De lo contrario, estaríamos ante un vicious regress o petitio principii, que haría del pensamiento la causa del símbolo y de este la causa del pensamiento. El símbolo no significa nada en sí mismo, son solo derivativamente representacionales (Kuczynski, 2005: 254). Sin embargo, comparte, con la CSP, el rasgo positivo. Es decir, tanto Derrida (representante de la CSP) ${ }^{5}$ como los psicólogos cognitivos, parten de una noción epistemológica en la cual lo real es lo dado, lo establecido ${ }^{6}$.

Los modelos mentales, entonces, encuentran siempre un referente en la realidad dada, que le da sustento cognitivo y epistemológico, el cual constituye su valor operativo.

\section{LOS MODELOS MENTALES ESPACIALES DENTRO DEL ENFOQUE COGNITIVISTA}

Iniciemos con la propuesta de William Langston y compañía. Ellos parten de la tesis que "la representación espacial es más topológica o funcional que euclidiana" (Langston, 1998: 247). Esta tesis tiene un grave problema, el cual radica en la indefinición de los términos "topológica", "funcional" e, incluso, "euclidiana". Por ejemplo, no sabemos si la palabra "topológica" refiere a su sentido etimológico, es decir, en el

Traducción y paréntesis cuadrados nuestros.

Derrida nos dice que Es pues verdadero a la vez que las cosas vienen a la existencia y la pierden al ser nombradas (1967: 107) Traducción nuestra. O sea, en el acto de habla, donde la conciencia se manifiesta, es el sitio en el cual el lenguaje se articula como un juego de significantes (de símbolos) que se encadenan entre sí, para construir un Sinn (sentido).

6 Volveremos sobre la positividad de la concepción de los modelos mentales, más adelante. Sin embargo, es necesario que el lector mantenga esto en mente, para amarrar las nociones de deducción, inducción y transducción que se desarrollarán posteriormente. sentido de "estudio del espacio", o si remite a la rama de la matemática que estudia las propiedades del espacio de una manera general y no-rígido; con lo cual realmente se alejaría de la geometría euclidiana que estudia las propiedades del espacio dentro de un espectro más particular y rígido. Dentro de esta vaguedad conceptual, es imposible determinar que indica, por cierto, la palabra "funcional", ya que, algo es funcional con respecto a alguna situación particular y aquí no es clara tal situación.

Tanto la geometría euclidiana, como la topología en sí son topológicas (parten de determinar una métrica para establecer las propiedades cuantitativas $y$ cualitativas del espacio, mediante el concepto de función ${ }^{8}$ ). Sin embargo, el carácter particular de la geometría euclidiana hace que su valor epistemológico se vea reducido ${ }^{9}$. O dicho de otro modo, la geometría euclidiana nos permite abordar los fenómenos como si estuviesen fijos, pero la realidad está en constante movimiento, por lo cual, es preciso una noción de espacio que abarque el movimiento ${ }^{10}$ como elemento $y$, por tanto, que permita incorporar el factor tiempo, haciendo del espacio un fenómeno de cuatro dimensiones. Esto lo veremos más adelante, en relación con las propuestas de Stephen Hawking.

No se puede entender la palabra "funcional", utilizada por Langston, fuera de su paradigma comportamental, o sea, como adaptable.

La función nos indica la forma en que se relacionan los elementos existentes en un espacio, dentro de la métrica definida para ese espacio, lo cual implica, a la vez, que para diferentes métricas, diferentes topologías.

9 De ahí que fenómenos como los hoyos negros y la relación de estos con el movimiento de la luz no pueden ser explicados por los principios de la geometría euclidiana, sino que se necesita la relatividad para hacerlo. Y dentro de esta visión relativista, la topología (por su carácter general) asegura una mayor confiabilidad a los resultados de la investigación.

10 Esta es la razón por la cual más adelante preferimos plantear el abordaje de los modelos mentales, dentro de un paradigma físico y no estrictamente matemático. 
Para Langston, los modelos mentales capturan las relaciones espaciales, dentro de un proceso que es similar al espacio tridimensional del espacio cotidiano (1998: 248). Es decir, el espacio que opera en la realidad concreta es el mismo que funciona en el proceso de representación del espacio textual, debido a que las "imágenes generadas por la descripción verbal pueden tener propiedades métricas" (Langston, 1998: 248) ${ }^{11}$.

La constitución del modelo mental espacial se establece mediante un tipo de inferencia llamado "anotamiento" (noticing), en donde los apuntadores (pointers) ${ }^{12}$ permiten evaluar la significación, cognitivamente hablando. En breves palabras, "El anotamiento significa que la relación entre los apuntadores es específicamente codificada y agregada a una información asociada en la memoria" (Langston, 1998: 249)13. En este sentido, los modelos mentales espaciales son topológicos o funcionales, por cuanto responden a un proceso que va de lo particular a lo general (o sea, un proceso inferencial) que permite solucionar los problemas de distribución espacial de los objetos y del posicionamiento del personaje en el espacio textual representado. Sin embargo, como el mismo Langston indicaba, estas representaciones espaciales tienen propiedades métricas; por tanto, propiedades cuantificables, como la simple noción de distancia.

En una oración de movimiento (move sentence) que describe a un personaje moviéndose desde una conocida locación a una nueva, en donde se pueda apreciar cómo los participantes (lectores) han construido una representación mental del espacio que es capaz de actualizarse, mediante y en el cambio de locación. Por esto, estos modelos no son representaciones estáticas (Langston, 1998: 257).

\section{Traducción nuestra.}

12 Los apuntadores son puntos de coordenadas que permiten colocar, en un orden específico, la información espacial recolectada.
Volviendo al anotamiento ${ }^{14}$, parece que Langston piensa que la base de un sistema euclidiano va ligada a la inferencia. Sin embargo, el problema no es si el modelo mental es deductivo $o$ inductivo. El problema real radica en si el modelo mental está relacionado con el tiempo $y$, si es así, cómo. Dentro de un esquema funcionalista ${ }^{15}$, las relaciones espaciales son no-euclidianas, sino que, más bien, responden a relaciones jerárquicas o topológicas que afectan la actuación (performance) (Langston, 1998: 260) ${ }^{16}$.

De igual modo, David J. Bryant ha estudiado los sistemas de representación espacial (SRE) en relación con el lenguaje. Bryant parte de la evidencia que "la gente puede construir adecuadas representaciones espaciales de ambientes transmitidos por descripciones verbales” (1992a). Es decir, según Bryant, la gente (los lectores) crea modelos mentales más que retener

Nuevamente, es difícil saber que entiende Langston por cada uno de los términos matemáticos que usa. Por ejemplo, el proceso inferencial del anotamiento parece ser el mecanismo que sostendría una noción de espacio euclidiano, en relación con la confirmación de los modelos mentales espaciales. No obstante, la geometría euclidiana es deductiva, ya que, ella deriva de leyes universales, o sea, de sus demostraciones o axiomas (Barker, 1965: 24-25). Entonces, cuando Langston liga el espacio euclidiano a, solamente, un proceso inferencial, caemos en cuenta que hay un error de concepto, por cuanto se separa la inducción de la deducción. Por ejemplo, la noción de cuadrado deviene históricamente de la necesidad de establecer los límites de un terreno de cultivo. Así, los egipcios midieron la tierra para asegurar la propiedad, en cuanto a sus límites, de las constantes inundaciones del Nilo. Luego, los griegos elevan el proceso $y$, por inferencia, crean deducciones rigurosas que explican el espacio y que, a la vez, sirven en la aplicación práctica. Filo-ontogenéticamente, deducción e inducción son las dos partes de un mismo proceso, en donde el ser humano se enfrenta a lo concreto.

15 Langston indica que los lectores codifican las relaciones espaciales de manera funcional (1998: 260).

16 Aquí es imposible no ver cierto pragmatismo epistemológico y nada de matemática. El planteamiento que hace Langston responde a un esquema behavorista, que es también cognitivo, pero que no pierde su mecanicismo. 
la estructura lingüística, en donde se mantienen las características físicas del espacio, como por ejemplo: la posición relativa y la distancia relativa. "Lo más importante, las características espaciales organizan la información dentro de los modelos y determinan la accesibilidad de la información desde modelos mentales" (Bryant, 1992a $)^{17}$. Por tanto, la relación espacio-modelos mentales opera en la aprehensión de la espacialidad textual representada.

Además, es importante rescatar el hecho que Bryant establece características espaciales como posición y distancia, las cuales se insertan dentro de un espectro cuantificable y tridimensional (representado bi-dimensionalmente, o sea, como plano ${ }^{18}$ que remite a la geometría euclidiana. No hay que olvidar que: "Sin embargo, es la representación discursiva en conjunción con el conocimiento general y las claves contextuales las que guían la construcción de los modelos mentales espaciales por el SRE" (Bryant, 1992a). O sea, las características espaciales (posición y distancia) se insertan dentro de un proceso inductivo-deductivo. Inductivo porque se parte de claves contextuales, es decir, de lo particular a lo general. Deductivo porque se elabora a partir de un cuerpo teórico-cognoscitivo previo, que podría remitirse, exempli gratia, al conocimiento matemático adquirido en la educación formal ${ }^{19}$.

En síntesis, Bryant indica que "el SRE crea representaciones que son modelos con aspectos físicos y funcionales del ambiente"

Traducción nuestra.

Son esquemas no-proposicionales (no-lingüísticos) compuestos de representaciones perceptuales que incluyen planos y puntos (Bryant, 1992a). Existe un isomorfismo entre la representación espacial y el espacio físico (Bryant, 1992b). Es decir, los lectores usan algún tipo de mapa representacional (map-like representation) para aprehender el espacio textual, antes que imaginar la escena descrita (Bryant, 1993).

19 "En general, la SRE organiza los modelos espaciales para establecer direcciones con algún especial estatus perceptual o comportamental más accesible desde la memoria" (Bryant, 1992a).
$(1992 a)^{20}$ con el fin de representar estas dimensiones y guiar el comportamiento en el espacio (Bryant, 1992b).

Claudia Brugman llama la atención sobre el papel del nivel léxico-gramatical. Según ella,

El sistema de traducción entre la representación lingüística y el SRE debe (...) tomar en cuenta las consideraciones desde el nivel más bajo (e.g., las categorías de las relaciones espaciales que son convencionalizadas en el lexicón) al más alto nivel (e.g., los modelos de inferencia resultantes del nivel discursivo) (Brugman, 1992) 21.

Es decir, la estructura léxica de cada lengua (referente a los adverbios de lugar, a las preposiciones y a las conjunciones) y las estructuras sintácticas, semánticas y pragmáticas conforman parte del proceso de modelación espacial.

Esto parece contravenir la posición de Bryant, quien afirma que son el conocimiento previo y las claves contextuales los elementos que conforman los modelos mentales espaciales establecidos por el SRE (Bryant, 1992a). Sin embargo, no se contraviene nada. La estructura del lenguaje condiciona, aunque no de manera absoluta, la aprehensión del espacio; pero el conocimiento (que implica una correspondencia entre realidad y lenguaje-pensamiento) también condiciona tal proceso.

Hasta ahora, nos hemos centrado en la discusión del papel del espacio en la conformación de los modelos mentales espaciales. No obstante, es hora de mirar un factor estrechamente correlacionado, o sea, el tiempo ${ }^{22}$.

Zwaan dirige sus reflexiones sobre el rol del tiempo en la comprensión textual, partiendo

\footnotetext{
20 Traducción nuestra.

21 Traducción nuestra.

22 Esto es fundamental, ya que, dependiendo de la concepción física que subyazca al análisis de los modelos mentales espaciales, tiempo y espacio pueden estar unidos o separados. Pero esto será analizado más adelante.
} 
de la hipótesis "que los mecanismos involucrados en la construcción de la situación-modelo, a partir del lenguaje, son derivados de los mecanismos de construcción de la situación-modelo en el mundo real" (Zwaan, sfe) ${ }^{23}$. Es decir, se intenta comprender cómo es construida la dimensión temporal en los modelos situacionales (modelos mentales) (Zwaan, sfe).

Zwaan afirma que la representación del tiempo en el lenguaje corresponde a una puesta en palabras, en una concatenación de eventos textuales, que son comparables con el fluir de los eventos en el mundo real (sfe). O sea, el lector intenta establecer una cronologización de los eventos mediante el aspecto verbal que guía tal concatenación (Zwaan, sfe).

Dentro de este marco, la narración establece una jerarquía de importancia en relación con los eventos narrados, más que el simple establecimiento de una secuencia temporal (Zwaan, sfe).

En resumen, Zwaan afirma que el proceso onto-filogenético utilizado para comprender el transcurrir de los eventos, es el mismo utilizado en la comprensión narrativa (textual) (Zwaan, sfe).

\section{LIMITACIONES DE LA RELACIÓN ESPACIO- APREHENSIÓN TEXTUAL EN EL ENFOQUE COGNITIVO}

En el punto anterior, hemos visto que la psicología cognitiva señala un paralelismo entre el modo que se establecen los modelos mentales espaciales de la realidad y la de los procesos de la aprehensión textual. Asimismo, se define un proceso de temporalización en la construcción de los modelos mentales, en donde los eventos adquieren un valor cronológico. No obstante, es preciso profundizar en las nociones de espacio $y$ de tiempo, que subyacen al análisis cognitivo (conductual).

Es claro que el espacio y el tiempo, dentro del enfoque cognitivo, están separados, ya que, el tiempo es, siguiendo a Zwaan, un elemento que ordena la secuencia de eventos sin afectar la organización espacial de la descripción literaria. En otras palabras, la alteración del tiempo no implica una alteración en el espacio y viceversa. Por tanto, cabe aquí preguntarse si el planteamiento del análisis de los modelos mentales espaciales en relación con la geometría es acertado o, de lo contrario, un error.

Si bien es cierto que el espacio dentro de la experiencia textual deviene un marco para la acción del personaje, también es verdad que el espacio es más que la experiencia positiva de un conjunto de posiciones particulares, que se relacionan con determinados y específicos objetos (e.g., la colocación de una silla, la dirección en que se mueve un personaje a lo largo de una calle, la descripción de un paisaje natural, etc.). O sea, el espacio trasciende la materialidad de las posiciones, por cuanto el tiempo siempre influye sobre él, debido a que, por ejemplo, en Los pasos perdidos de Alejo Carpentier, es claro como (y de manera muy metafórica) cuando el personaje principal pasa de Europa al Amazonas, se produce una alteración en la experienciación de tales espacio-temporalidades. Esto por cuanto el tiempo de la ciudad europea responde a un tiempo estructurado y codificado (el reloj es clara manifestación de esto). Mientras que el tiempo en el Amazonas está más ligado al ritmo de la vida cotidiana $y$, por consiguiente, está menos codificado. Por ende, la asunción de la relación espacio-tiempoliteratura se inserta más dentro de una perspectiva física que geométrica. Expliquemos con detalle esta afirmación.

Dentro del análisis cognitivista de los modelos mentales espaciales, encontramos una característica clave para explicar cuál es el meollo del asunto, o sea, la ya mencionada separación tiempo-espacio. Esta separación refiere a la física mecanicista, en donde el tiempo es absoluto (entiéndase constante e inalterable) y el espacio es un conglomerado de tres dimensiones (altura, anchura y profundidad). Este espacio tridimensional que es no-afectado por el tiempo, es el espacio de la geometría euclidiana, en la cual las características cuantificables son fundamentales en la comprensión del espacio. Exempli gratia, la noción de distancia, utilizada por Langston o Zwaan, es un concepto 
mesurable y representado mentalmente como un plano o eje de coordenadas bidimensional ${ }^{24}$. En el diario vivir, nosotros nos valemos de la geometría euclidiana en todo momento, cuando cruzamos la calle y calculamos la velocidad a la que viene un automóvil o cuando debemos movernos entre una serie de objetos dispuestos en un cine, nuestra casa, etc.

Es decir, en nuestros desplazamientos cotidianos el tiempo permanece inalterable, por cuanto la posición que se ocupe en un determinado espacio, no altera la percepción-conducta del tiempo mismo. Por ejemplo, si en una escena un personaje se desplaza dentro de un sitio, en donde hay otros personajes; entonces, independientemente de que cada uno de esos personajes, percibirá el movimiento del personaje que se desplaza dentro de una misma unidad de tiempo, la cual será mesurable en minutos o segundos. Esto prueba el valor absoluto del tiempo, en ambientes de este tipo ${ }^{25}$.

Entonces, la deducción y la inducción que opera en los modelos mentales espaciales, según la propuesta cognitiva analizada, parten de un conjunto de conocimientos previos sobre el espacio que permiten aprehender el espacio en todos los momentos posibles, o parten de una serie de experiencias particulares que se generalizan para crear un concepto de espacio válido en todo momento. En todo caso, parece ser que esta noción de espacio de los modelos mentales implica una absolutización del tiempo; ergo, el enfoque cognitivo reproduce un esquema físico mecánico o newtoniano.

El desarrollo de la física moderna, sin embargo, nos enseña que "Debemos aceptar que el tiempo no está completamente separado e independiente del espacio, sino que por el contrario se combina con él para formar un objeto llamado espacio-tiempo" (Hawking, 1988: 43-44). Entonces, si el tiempo y el espacio son una unidad indisoluble, $y$, además, los modelos mentales

Esto es lógico, ya que, es imposible introducir en nuestra mente el espacio en su profundidad; luego, la representación es siempre plana.

Esta noción de tiempo y espacio remite al pensamiento aristotélico-newtoniano, en donde el tiempo $y$ el espacio se encontraban totalmente separados e independizados uno del otro (Hawking, 1988: espaciales (con los que se aprehende el texto) son los mismos para el espacio real y para el espacio representado; por consiguiente, son todos los modelos mentales determinados por la fisicidad del proceso de aprehensión textual. Esto adquiere fundamento en la segunda ley de la termodinámica, o sea, el principio de entropía que dice que "Nuestro sentido subjetivo de la dirección del tiempo, la flecha psicológica del tiempo, está determinado por tanto dentro de nuestro cerebro por la flecha termodinámica del tiempo" (Hawking, 1988: 194). Es decir, podemos recordar el pasado, pero no podemos recordar el futuro (Hawking, 1988: 200).

Según la segunda ley de la termodinámica, nuestro cerebro introyecta la relación tiempo-espacio como unidad indisoluble. Entonces qué pasa con la concepción de los modelos mentales establecida por la psicología cognitivo-conductual. Como lo hemos dicho, el enfoque cognitivista tiene serios errores en el nivel de la conceptualización matemática que usan. Pero, además, el substrato positivista que fundamenta tal enfoque crea un horizonte epistemológico bastante chato, por lo que el espacio no es concebible más allá de su inmediatez, la cual es producto de haber olvidado la dimensión física del fenómeno analizado, el espacio.

En síntesis, los resultados del enfoque cognitivista tienen éxito al afirmar que hay algún tipo de isomorfismo entre la forma que los modelos mentales aprehenden la realidad $y$ el texto. No obstante, este enfoque falla rotundamente en la explicación del fenómeno, ya que, no es capaz de romper con la vieja separación del tiempo y del espacio, ya superada por la física moderna. Es preciso entonces repensar los modelos mentales más allá de su valor positivo y de su anclaje deductivo-inductivo, que lo cierran dentro de una temporalidad cerrada, en donde el análisis presente solo tiene la opción de retomar el pasado, pero nunca de prever el futuro o, dicho de otra forma, de asumir la constitución de modelos mentales tempoespaciales anclados en la negatividad $^{26}$.

26 El concepto de negatividad lo hemos explicado en: (Alfaro, 2005b y Alfaro, 2006). 


\section{LOS MODELOS MENTALES TEMPOESPACIALES}

Un modelo mental tempoespacial es un esquema cognitivo que permite aprehender el fenómeno literario, dentro de la unidad de tiempo-espacio y en relación con el principio de entropía, lo cual implica que hay una relación pasado-presente-futuro ${ }^{27}$, que es aprehensible mediante la dialéctica negativa, a través de un proceso de transducción ${ }^{28}$. Es decir, se analizan las formas de representación pasadas (y contemporáneas) desde el presente, con el fin de imprimirle al contenido la dirección deseada, en busca de nuevas futuras formas.

Mediante la aplicación de la transducción, nos es permitido concebir la praxis dentro de una realidad situada más allá de la identidad positiva del Ser y, entonces, comenzar a crear una realidad desde la conciencia de la unidad de identidad $y$ negatividad. Con esto en mente, podemos introducir, dentro de la conformación social de los modelos mentales, una de las dos características del movimiento olvidada por el enfoque cognitivista, es decir, la noción de cambio $^{29}$.

Con los modelos mentales tempoespaciales, seremos capaces de acceder a una realidad

El tiempo está desplegado en su completitud, tanto pasado, como futuro están colocados juntos; o sea, el tiempo ni transcurre ni fluye, lo cual no implica que el flujo del tiempo sea irreal (Davies, 2000a: 9). En otras palabras, en relación al espacio, aquí y allá siempre están desplegados independientemente de si nos encontramos en cualquiera de estos dos puntos; así el pasado y el futuro dependen de nuestra posición y nuestro movimiento en relación con la velocidad de la luz.

La transducción es la lógica del objeto posible (Lefebvre, 1976: 18).

29 La noción de movimiento implica tanto el desplazamiento mecánico y el cambio (Bitsakis, 1975: 94). Evidentemente, dentro del enfoque cognitivista, solo se ha hecho hincapié en la parte del desplazamiento mecánico, nunca en la noción de cambio, que de hecho conlleva la noción de cambio social, la cual no es muy compatible con un esquema positivo de gran auge en las tierras anglosajonas. dinámica, en la cual objeto y sujeto tengan, realmente, una relación dialéctica. Estos devendrán un espacio situado en un punto más allá de la interpretación textual, sino situados en el espacio de construcción práxica de las posibilidades de escritura ${ }^{30}$.

Es preciso entender que los modelos mentales espaciales o tempoespaciales responden a la lógica de los procesos de socialización $y$ que, en tanto procesos onto-filogenéticos, son construibles y reconstruibles socio-históricamente. Por tanto, no es imposible crear formas de pensar que restablezcan el equilibrio de la unidad tiempo-espacio, que ha sido colapsado por la lógica de nuestra sociedad clasista, en donde un grupo pretende eternizarse como élite, mediante la manipulación del espaciotiempo ${ }^{31}$. Ejemplo de esto es el mismo enfoque cognitivista, que produce una investigación que lleva en sí el rompimiento de la unidad tiempoespacio. Luego, sus resultados se publican y son asumidos por las casas de enseñanza. Los resultados de la investigación devienen, entonces, parte del fenómeno pedagógico dentro de los aparatos ideológicos del Estado (Althusser) y se insertan, ahí directamente, en los procesos de socialización de una sociedad de clases. En consecuencia, el alumno aprende la teoría, pero también es expuesto al modelo de socialización subyacente al cuerpo teórico.

30 El rol del lector no es solo interpretar a través de los procesos de legitimación de la crítica literaria o de la intuición de su constitución identitaria, sino que su rol es introducir el texto en su vivencia cotidiana para destruirlo, en un proceso que ofrecerá nuevas posibilidades de escritura. Es decir, la forma (el texto) se inserta en el devenir del contenido (la praxis) para plasmarse en nuevas formas. El texto que permanece en el tiempo es ideología. Una ideología que se legitima como la expresión formal que sostiene los procesos identitarios, dentro de un afán historicista de la crítica literaria. La buena literatura viene, cumple su función y desaparece (la historia atestigua esto, su ausencia lo justifica); no se anquilosa como historicismo.

31 Recordemos que El espacio y el tiempo no sólo afectan, sino que también son afectados por todo aquello que sucede en el universo (Hawking, 1988: 56). 
Parte de la tarea que le asignábamos a la sociología dialéctica de la literatura ${ }^{32}$ es determinar los procesos intra-extra textuales que confluyen en la organización de los esquemas de espacio-tiempo, tanto en los textos particulares, como en los procesos de legitimación de la crítica literaria. Es decir, esta sociología se dirige, primeramente, al esclarecimiento de las nociones antropológicas que se ocultan en los procesos oficiales (establecidos en la cotidianidad o por los aparatos ideológicos del Estado) y que impiden, momentáneamente, acceder a nuevos contenidos antropológicos ${ }^{33}$. Todo con el fin de establecer los elementos pertinentes para una pedagogía, que responda a nuestros procesos históricos y sociales, $y$ que devenga el elemento fundamental de liberación de las metrópolis del capitalismo globalizante.

La búsqueda de las constantes representacionales del tiempo y del espacio, dentro de la literatura, en particular, y en la cultura, en general, nos permitirá establecer los parámetros en que tales categorías son manipuladas, para sostener lo establecido. Esto implica romper con la lógica clasificatoria de la filología clásica ${ }^{34} y$, así, tener acceso a una Literaturwissenschaft creativa, crítica e insertada de manera franca en el desarrollo de nuestras sociedades. En otras palabras, una Literaturwissenschaft desarticulada de todas las formas de existencia de la noción de entender la literatura como "las bellas letras". Por tanto, es el principio para alejarnos de la paleontología literaria que vive de intentar traer a la vida los fósiles formales y formalizados de la historiografía literaria.

Nos referimos al análisis dialéctico de la relación individuo-sociedad, a través de la asunción de la literatura como mediación de esa relación, en el marco de una integración social dada en el conflicto de la lucha de clases del capitalismo globalizante (Alfaro, 2005a: 76).

33 El lector puede encontrar una introducción a las antropologías literarias, en: (Alfaro, 2007a).

Con el término filología clásica, definimos no sólo la filología greco-latina, sino también todas las corrientes de análisis literario, que se anclan en el análisis de la forma; por ejemplo: los estudios culturales, el estructuralismo, las semióticas, etc.
Es imposible entender racionalmente cómo textos alejados en el tiempo y el espacio, producto de sociedades y modos de producción que yacen bajo tierra, sigan dominando gran parte del escenario literario. Textos como $\mathrm{La}$ Odisea de Homero (en el contexto mundial) o Cuentos de Magón (en el caso costarricense) se rescatan constantemente dentro de las publicaciones críticas, con el único fin de permitir al crítico mostrar su conocimiento al respecto de tales textos, pero sin un criterio real que indique por qué hoy seguimos leyendo tales textos. Pseudoargumentos sobran para justificar su lectura, desde criterios estéticos hasta aquellos que abogan por su importancia en los procesos identitarios. Ninguno de ellos tiene una base real-racional que les permita sostenerse. Los criterios estéticos, per esempio, nos llevan a la cuenta de qué son realmente ellos, la imposición de una sociedad (Genette) o el producto de una sensación de placer o displacer que experimentamos ante el objeto (Kant). En cualquiera de estos casos, la base epistemológica es frágil, ya que, su realidad depende o de un no-acordado contrato social que acepta equis texto como bello, de gran calidad, etc.; o de una propiedad que no pertenece al objeto, sino al sujeto que lo experimenta como placentero. Por ende, ambos criterios responden a una forma, que se pretende independiente del movimiento del contenido ${ }^{35}$.

El modelo mental tempoespacial deviene la posibilidad de pensar el texto lejano, por ser la mediación (Vermittlung) entre él y el texto presente. Es la articulación, como pensamiento, de la continuidad y la discontinuidad, de lo finito $y$ lo infinito; o sea, cada texto es un punto en la recta (flecha) del tiempo, que, como toda existencia, es finita en su forma presenta e infinita dentro del proceso de la historia (Geschichte).

El lector puede encontrar una reflexión más sistematizada al respecto de la forma y el contenido, en nuestro artículo "Forma y movimiento en los estudios literarios" (Alfaro, 2007b), el cual está en proceso de publicación, en esta misma revista, en el momento de escribir estas líneas. También, se puede consultar (Alfaro, 2006) en donde, al respecto de la dialéctica lefebvriana, analizamos la relación gnoseológica de las categorías de forma y contenido. 


\section{CONCLUSIÓN}

El desarrollo del estudio de los modelos mentales espaciales dentro del enfoque psicológico cognitivista, a pesar de sus yerros, nos muestra que existe un nexo entre la forma en que asumimos el espacio real y el espacio representado. Por otro lado, el desarrollo actual de la física nos permite abordar el problema de los modelos mentales espaciales de manera tal que podamos articular su estudio y desarrollo con la noción de un espacio-tiempo como unidad.

Hoy hemos podido plantear la antítesis de los modelos mentales espaciales desarrollados por los cognitivistas, es decir, los modelos mentales tempoespaciales, con los cuales podemos no sólo analizar los textos dados, sino también los textos posibles en tanto posibilidades de escritura. A la vez, se desacraliza la idea de entender el texto como forma, asumiéndolo como parte integral de la praxis desplegada onto-filogenéticamente en el pasado-presentefuturo del Ser real (material).

Finalmente, podemos afirmar que la representación del espacio dentro del texto se inserta dentro de un modelo mental caracterizado como un espacio euclidiano, el cual se inserta a la vez dentro de una noción físicomecánica (física newtoniana) la cual responde a un proceso de disonancia cognitiva que niega la indisoluble relación tiempo-espacio, más ligada a la física relativista, y que, por tanto, tal disonancia se establece como un medio positivo de control social.

\section{BIBLIOGRAFÍA CONSULTADA}

Alfaro Vargas, Roy. "Relación literaturasociedad. Una aproximación teórica”. Revista de Ciencias Sociales 108. Universidad de Costa Rica. 2005a: 71-78.

- "Sociología dialéctica de la literatura: cuestión de método". Revista de Ciencias Sociales 109-110. Universidad de Costa Rica. 2005b: 151-156.

"La sociología crítica de Henri Lefebvre". Revista de Ciencias Sociales
113-114. Universidad de Costa Rica. 2006.

. "Antropologías literarias: prolegómenos a la praxis textual". Praxis: Revista de la Escuela de Filosofía 59, enero-junio. Universidad Nacional. 2007a: 7-17.

. "Forma y movimiento en los estudios literarios". Revista de Ciencias Sociales. 117-118. Universidad de Costa Rica. 2007b.

Althusser, Louis. Ideología y aparatos ideológicos del Estado. Sétima reimpresión. México: Ediciones Quinto Sol, 1987:86.

Barker, Stephen F. Filosofía de las matemáticas. Traducción: Carlos Moreno Cañadas. México: UTEHA, 1965: 172.

Berger, Peter y Luckmann, Thomas. La construcción social de la realidad. Traducción: Silvia Zuleta. Buenos Aires: Amorrortu Editores, 1968: 236.

Bitsakis, Eftichios. Física contemporánea y materialismo dialéctico. Traducción: José Fernández Valencia. México: Ediciones de Cultura Popular, 1975: 268.

Brugman, Claudia. "Spatial Cognition: the Perspective from Theoretical Semantics". Psycoloquy 3 (45). 1992. En: <www.cogsci. ecs.soton.ac.uk/cgi/psyc/newpsy? space.6> (recuperado el 20 de septiembre de 2007).

Bryant, David J. "A Spatial Representation System in Humans”. Psycoloquy 3 (16). 1992a. En: <www.cogsci.ecs.soton.ac.uk/ cgi/psyc/newpsy?3.16> (recuperado el 20 de septiembre de 2007).

"More on the Spatial Representation System". Psycoloquy 3 (44). 1992b. En: $<w w w . c o g s c i . e c s . s o t o n . a c . u k / c g i / p s y c /$ newpsy?space.5> (recuperado el 20 de septiembre de 2007). 
. "Lexical Contributions to Spatial Representation”. Psycoloquy 3 (51). 1992c. En: <www.cogsci.ecs.soton.ac.uk/ cgi/psyc/newpsy?space.9> (recuperado el 20 de septiembre de 2007).

. "Multiple Frames of Reference". Psycoloquy 4 (13). 1993. En: <www. cogsci.ecs.soton.ac.uk/cgi/psyc/ newpsy?space.13> (recuperado el 20 de septiembre de 2007).

Carpentier, Alejo. Los pasos perdidos. $2^{\text {a }}$ edición. San José, Costa Rica: EDUCA, 1998: 269.

Davies, Paul. "La flecha del tiempo". Investigación y ciencia 314, noviembre. 2002a: 8-13.

"La máquina del tiempo". Investigación y ciencia 314, noviembre. 2002b: 16-21.

Derrida, Jacques. L'écriture et la différance. Paris: Éditions du Seuil, 1967: 440.

Einstein, Albert. La relatividad. Traducción: Ute Schmidt de Cepeda. México: Grijalbo, 1991: 204.

Ezzell, Carol. "El tiempo cultural". Investigación y ciencia 314, noviembre. 2002: 42-43.

Ferstl, Evelyn C.; Rinck, Mike; and Cramon, D. Yves von. "Emotional and Temporal Aspects of Situation Model Processing during Text Comprehension: An EventRelated fMRI Study". Journal of Cognitive Neuroscience 17 (5). Massachusetts Institute of Technology. 2005: 724739. En: <http://www-psych.stanford. edu/ lera/273/rinck-jcn-2005.pdf > (recuperado el 30 de junio de 2007).

Garnham, Alan. "Space: the Final Frontier?". Psycoloquy 4 (30). En: <www.cogsci. ecs.soton.ac.uk/cgi/psyc/newpsy?4.30> (recuperado el 27 de septiembre de 2007).

Genette, Gérard. "Relaciones axiológicas". Revista de Occidente 213, febrero. Traducción: A.T. 1999: 9-23.

Hawking, Stephen W. Historia del tiempo. Del Big Bang a los agujeros negros. $3^{a}$ edición. Traducción: Miguel Ortuño. México: Editorial Crítica, 1988: 248.

Homero. La Odisea. Buenos Aires: EspasaCalpe, 1951: 258.

Kafka, Franz. La metamorfosis. Traducción: Jorge Luis Borges. Buenos Aires. Editorial Losada, 1973: 137.

Kant. Crítica del juicio. $2^{\text {a }}$ edición. Traducción: José Rovira. Buenos Aires: Editorial Losada, 1968: 344.

Kasner, Edward y Newman, James. Matemáticas e imaginación. Tercera edición. Traducción: J. Caldeiro Ricoy. México: Compañía Editorial Continental, 1959: 397.

Kuczynski, John-Michael. "The concept of a symbol and the vacuosness of the symbolic conception of thought". Semiotica 154 (1/4). 2005: 243-263.

Langston, William; Kramer, Douglas C.; and Glenberg, Arthur M. "The Representation of Space in Mental Models derived from Text". Memory and Cognition 26 (2). 1998 : 247-262. En: <http://www.psychonomic.org/search/ view.cgi?id=1984> (recuperado el 27 de julio de 2007).

Lefebvre, Henri. Lógica formal, lógica dialéctica. $4^{a}$ edición. Traducción: María Esther Benitez. México: Siglo Veintiuno Editores, 1976: 350. 
Éléments de rythmanalyse. Introduction à la connaissance des rythmes. Paris: Éditions Syllepse, 1992: 120.

Macho Stadler, Marta. "¿Qué es la topología?” Sigma 20, febrero. 2002: 63-77.

Magón (seud.). Cuentos. San José, Costa Rica: Editorial Costa Rica, 1980: 408.

Rojas Rojas, Alberto. "Espacio y tiempo en las sociedades globales según Manuel
Castells". Praxis: Revista de la Escuela de Filosofía 57. Universidad Nacional de Costa Rica, 2004: 59-64.

Zwaan, Rolf A.; Madden, Carol J.; and Stanfield, Robert A. (s.f.e.). Time in Narrative Comprehension: A Cognitive Perspective. En: <http://freud.psy.fsu.edu/ zwaan/ time_in_narrative.pdf $>$ (recuperado el 30 de junio de 2007). 